\title{
Rheological Properties of Xanthan Gum and Polyacrylamide Mixture in Inorganic Salt Solutions
}

\author{
Huanan Liu ${ }^{1,2}$, Jinghua $\mathrm{Wu}^{1}$, Yichen Zhang ${ }^{1}$, Wei Wang ${ }^{1}$, Yanan Chen ${ }^{1}$, Qiuling Lang ${ }^{1}$, Xiaolong Gao ${ }^{1}$, Jianshan Song ${ }^{1}$, \\ Xiangjun $\mathrm{Pei}^{2 *}$ \\ ${ }^{1}$ School of Prospecting and Surveying Engineering, Changchun Institute of Technology, Changchun 130021, China \\ ${ }^{2}$ State Environmental Protection Key Laboratory of Synergetic Control and Joint Remediation for Soil \& Water Pollution, \\ College of Ecology and Environment, Chengdu University of Technology, Chengdu 610059, China
}

Corresponding Author Email: peixiangjun2012@cdut.cn

https://doi.org/10.18280/ijht.390436

Received: 8 April 2021

Accepted: 26 June 2021

\section{Keywords:}

xanthan, polyacrylamide, rheology

\begin{abstract}
PAM is a good flocculant, and XG is a good tackifier. When the two solutions are mixed, the PAM/XG mixed solution has good viscosity and can be used as a foam stabilizer in foam drilling fluids, and it can also be used as a coupling agent for sand-fixation serous fluid. When metal cations enter the PAM/XG system, it will destroy the overall rheology and stability of the PAM/XG system. This article uses the DV3T-LV rheometer to test the rheology of different types of PAM (HPAM/CPAM/AMPAM/NPAM) and XG mixed solutions, and get the optimal test ratio. Then add various metal cations $\left(\mathrm{Na}^{+}, \mathrm{K}^{+}, \mathrm{Ca}^{2+}\right.$, $\mathrm{Mg}^{2+}$ ) to the mixed solutions of HPAM/XG, CPAM/XG, AmPAM/XG, NPAM/XG respectively. We know divalent metal cations have greater impacts on the shear stress of the PAM/XG composite system, which is likely to cause its shear dilution. Finally, through mechanism analysis, it is concluded that it provides a good preliminary foundation for the research of foam drilling fluid and sand-fixation serous when the metal ion destroys the molecular structure of the PAM/XG composite system.
\end{abstract}

\section{INTRODUCTION}

Polyacrylamide (PAM) is a water-soluble linear polymer material that polymerized by monomer polyacrylamide $[1,2]$. PAM is a water-soluble polymer, insoluble in most organic solvents, has good flocculability and can reduce the friction between liquids [3]. Polyacrylamide flocculants can be divided into nonionic polyacrylamide (NPAM), anionic polyacrylamide (HPAM), cationic polyacrylamide (CPAM) and amphoteric polyacrylamide (AmPAM) according to their charge properties [4]. The structural unit of polyacrylamide contain amide groups, which are easy to form hydrogen bonds [5]. Therefore, it has good water solubility, and has the advantages of less dosage and fast flocculation, can be used as flocculant and thickener. Polyacrylamide is mainly used as flocculant and widely used for the petroleum industry, oil production, drilling mud, textile industry and paper industry [6-8].

PAM is a linear water-soluble polymer. Because of the flexibility of its molecular chain and the variability of molecular shape (chain conformation), as well as its high polarity, it is easy to form hydrogen bond and highly reactive amide group [9], which makes polyacrylamide have many extremely valuable performance, and it is easy to obtain various modifications of branch or network structure by grafting or cross-linking [10]. XG polymer is also called Xanthan gum, it is a polymeric, chain-like polymers polymer formed by the desert fungus acting on carbohydrates, and it's relative molecular weight can be up to $5 \times 10^{6}$, and soluble in water [11]. Xanthan gum (XG) contains strong polar groups such as - $\mathrm{COO}-,-\mathrm{OH}$, and other strong polar groups. And the charged side-chains of the molecule are wrapped backwards around the main chain [12]. In the ordered state, the main chain and the side chain form a double helix structure by hydrogen bonding. These spiral structures also maintain their stability by electrostatic force and steric effect. At the same time, when the double helix structure is combined by covalent bond, it can also form a regular network of spiral copolymers. These network structures make the molecules have strong rigidity, so that make the molecular chain of xanthan gum in aqueous solution stretched, and the fluidity of aqueous solution can be well controlled [13].

Therefore, xanthan gum solution has good tackifying properties. Polyacrylamide and xanthan gum is good foam stabilizer in the use of drilling fluid [14]. They are also good flocculants and tackifiers in the use of sand fixation. Because the natural water containing different metal ions, the amount of ions in the water will inevitably affect the properties of polyacrylamide and xanthan gum. Therefore, we respectively mixed four kinds of polyacrylamide (NPAM, CPAM, AmPAM and HPAM) with XG to form supramolecular polymer, to research the effect of metal ions on the rheological properties of the mixed solution and its mechanism by DV3TLV rheometer, so that we can clearly understand the effect of metal ions on the rheology of PAM/XG mixed solution.

\section{EXPERIMENTAL}

\subsection{Materials}

XG was purchased from Handong Shenglong Chemical 
Industry (China). PAM was purchased from Henan Hengsheng Water purification materials Co., Ltd. (China). All other reagents are of analytical grade. The $\mathrm{KCl}$ (A.R.), $\mathrm{NaCl}$ (A.R.), $\mathrm{MgCl}_{2}$ (A.R.) and $\mathrm{CaCl}_{2}$ (A.R.) were purchased from Tianjing Fuchen Chemical Reagents Factory (China). Freshly distilled water was used in all solution preparations.

\subsection{Solution preparation}

Take a certain amount of distilled water in proportion, and add polyacrylamide to distilled water slowly. The subsequent preparation conditions are as follows: stirring speed is 800 RPM, stirring time is 30 minutes. Then add xanthan gum to the prepared polyacrylamide solution, and stir at high speed to make a mixed solution. The solution of metal ions should be dissolved in distilled water separately, then add it to the mixture of polyacrylamide and xanthan gum after it is dissolved. The prepared solution needs to be placed 24 hours stably to elminate residue and air bubbles. Before the experiment, the mixed solution of $\mathrm{PAM} / \mathrm{XG}$ that containing different concentrations of inorganic salt metal ions should be stirred at low speed for 5 minutes after removing the bubbles, then conduct rheological tests to ensure the accuracy of the experiment.

\subsection{Methods and measurements}

The rheological measurement dates of all the samples were carried out by a DV3T-LV rheometer (Brookfield Inc, American).

\section{RESULTS AND DISCUSSION}

\subsection{The effect of different concentration ratios on its rheology}
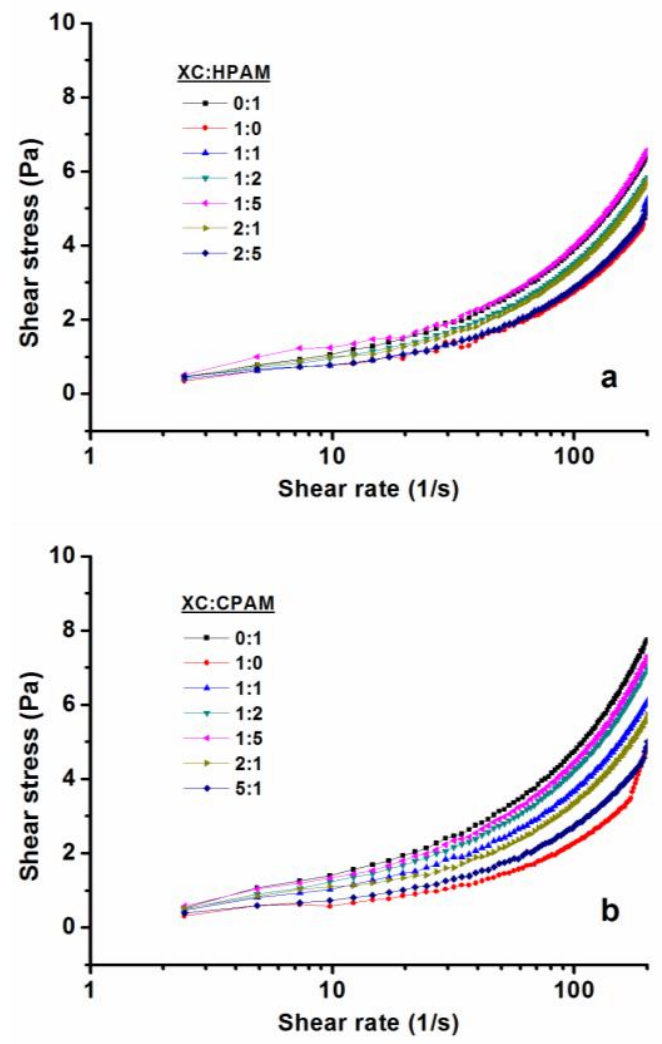
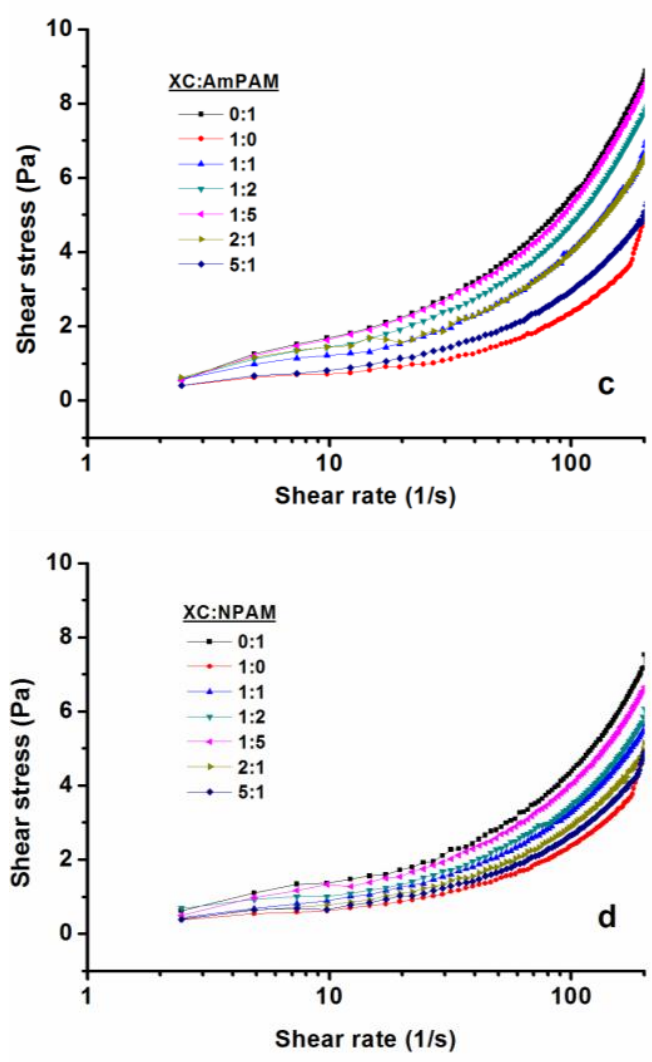

Figure 1. Shear stress versus shear rate for PAM and XG mix solutions at various polymer concentrations tested at different ratios

Select a solution with a certain concentration of $0.02 \%$, and divide the xanthan gum XG and polyacrylamide PAM into proportions of $1: 0,1: 2,1: 5,5: 1,1: 1,2: 1,0: 1$.

Figure 1 shows us the curve of shear stress and the curve of shear rate of XG and PAM in different scales. It can be seen from Figure 1 that anionic polyacrylamide and xanthan gum show little difference in rheological properties at different proportions, the combination of polyacrylamide and xanthan gum can enhance the rheological properties of the solution. As can be seen from Figure 1(a, b, c and d). The rheology of xanthan gum solution is very poor, the rheological property of polyacrylamide solution is much better than the mixed solution which mixed with xanthan gum. From Figure 1, it can be concluded that the ratio of rheological property is XG/PAM: $0: 1>1: 5>1: 2>1: 1>2: 1>5: 1>1: 0$. It shows that the ratio mixing system not only has a higher viscosity synergistic effect, but also has better salt-resistance than single solution. Therefore, we chose $\mathrm{XG}: \mathrm{PAM}=1: 1$ as the basic research on the rheological properties of metal ions on the mixed solution.

\subsection{Effect of concentrations of different inorganic salts on steady shear viscosity}

3.2.1 The influence of inorganic metal ions on the rheology of HPAM and XG

In this experiment, we selected four metal ions and added them to the HPAM/XG mixed solution. Their concentrations are $0.05 \%(500 \mathrm{mg} / \mathrm{L}), 0.1 \%(1000 \mathrm{mg} / \mathrm{L}), 0.5 \%(5000 \mathrm{mg} / \mathrm{L})$, $1.5 \% \quad(15000 \mathrm{mg} / \mathrm{L}), \quad 2.5 \% \quad(25000 \mathrm{mg} / \mathrm{L}) \quad$ and $\quad 3.5 \%$ $(35000 \mathrm{mg} / \mathrm{L})$, other components remain unchanged. As can be seen from Figure $2 a, 2 b$, with the increase of $\mathrm{Na}^{+}$and $\mathrm{K}^{+}$ion concentration, the shear stress also decreases and tends to be 
stable at about $2.5 \%$. And it can be seen from Figure $2 \mathrm{c}$ and $2 \mathrm{~d}$, at high shear rates, $\mathrm{Ca}^{2+}$ and $\mathrm{Mg}^{2+}$ ions at the concentration of $0.05 \%$ have a great influence on the shear stress of the mixed solution, the impact range can be up to $50 \%$.Meanwhile, with the increase of $\mathrm{Ca}^{2+}$ and $\mathrm{Mg}^{2+}$ ion concentration, the shear stress remains unchanged, It can also be seen from Figure 2 that when the concentration of univalent metal ions increases to $2.5 \%$, the influence on the rheological properties of the mixed solution remains the same as that of divalent metal ions at $0.05 \%$. So It can be seen that the influence of divalent metal ions on HPAM and XG solutions is greater than that of monovalent metal ions.

It can be seen from Figure 3 that the groups between HPAM and $\mathrm{XG}$ are connected into a spatial network structure in the form of hydrogen bond, which makes the molecular chain of HPAM / XG system very stretched in aqueous solution and can well control the fluidity of aqueous solution. When there are univalent metal cations $\left(\mathrm{Na}^{+} 、 \mathrm{~K}^{+}\right)$in the mixed solution, cation inhibits the electrostatic repulsion between carboxyl ions in polyacrylamide molecules, then the hydrogen bond between HPAM and XG is broken, making the polyacrylamide molecule crimp, the higher the degree of hydrolysis (the higher the content of carboxyl), the more serious the molecular crimp of polyacrylamide in saline water, the worse the thickening property. When salt is added to the HAPM solution, whether it is monovalent cation or divalent cation, the viscosity of the solution will be reduced. Among them, divalent cations such as $\mathrm{Ca}^{2+}$ and $\mathrm{Mg}^{2+}$ have greater influences on the viscosity of HAPM solution than monovalent cations such as $\mathrm{Na}^{+}$and $\mathrm{K}^{+}$. Especially when high-valent metal cations contact with it, the polar groups on the molecules will combine with counter ions, then the molecules lose their internal repulsion and shrink the coils, and reduce the hydrogen bonds between the polymer bonds and the interacting water molecules [15]. The network structure of extended macromolecules is destroyed, resulting in the reduction of effective diameter of molecules in solution and the decrease of solution viscosity [9]. The reason for this phenomenon is that divalent ions such as $\mathrm{Ca}^{2+}$ and $\mathrm{Mg}^{2+}$ can interact with carboxyl ions so that HAPM from the solution can be precipitated under certain conditions, and result in a significant decrease in the viscosity of the HAPM/XG mixed solution.
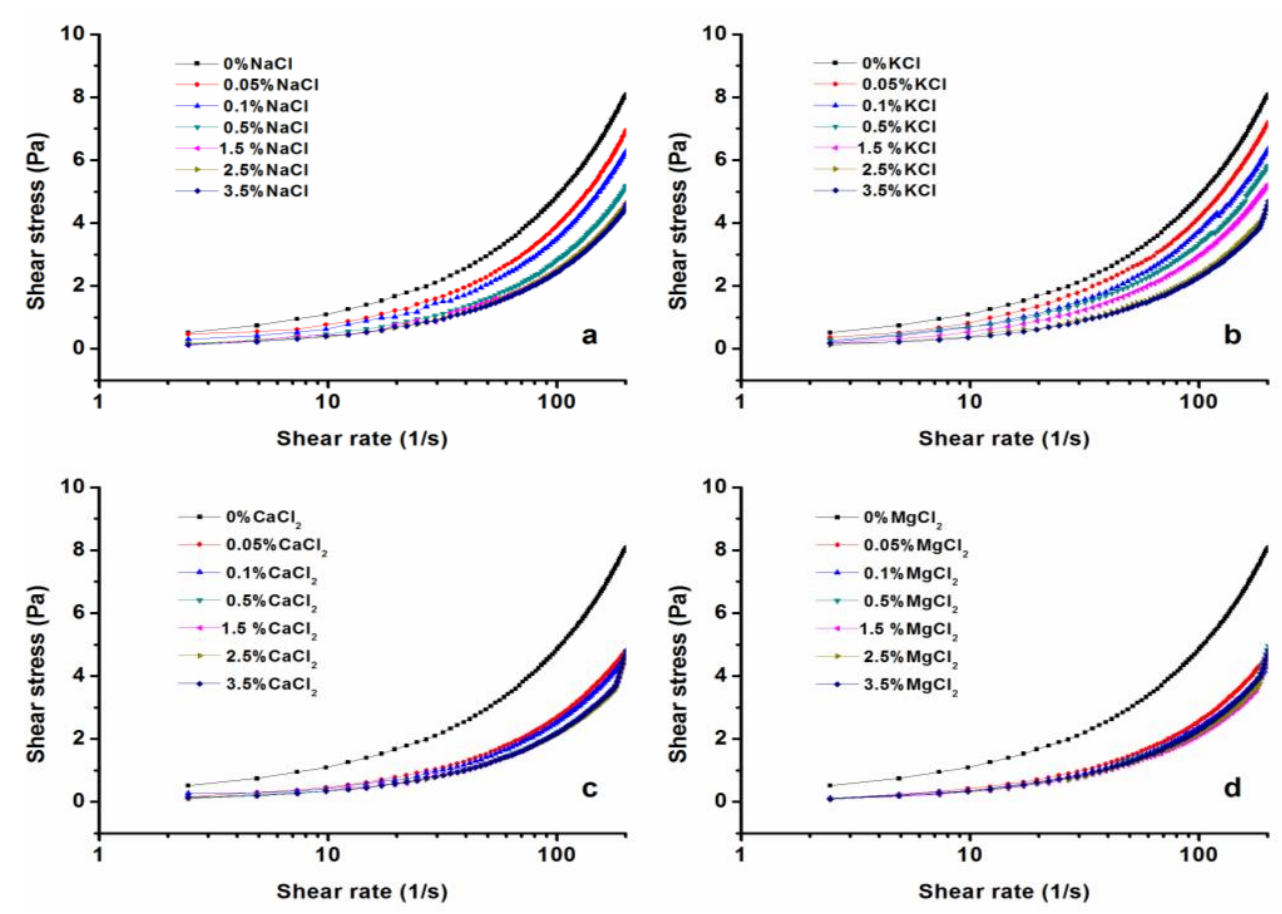

Figure 2. Effect of Inorganic Metal Ions on Rheology of Mixed Solution of HPAM and XG

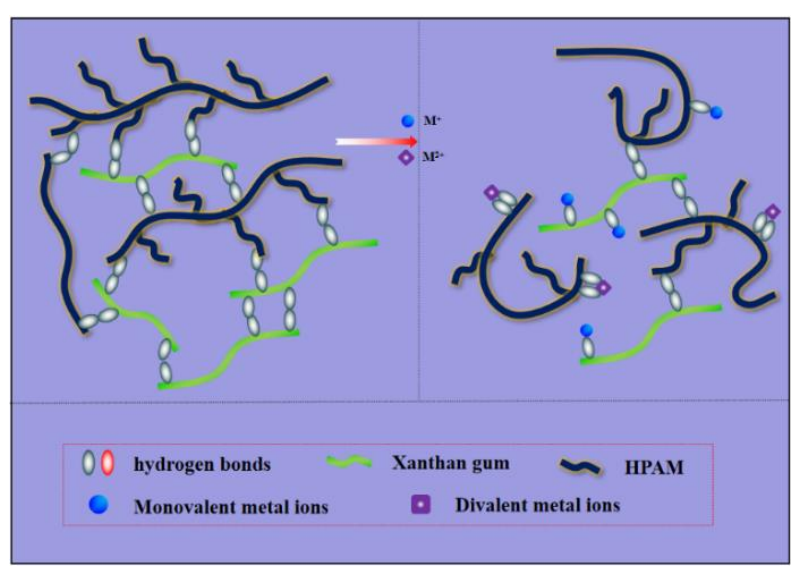

Figure 3. The mechanism of metal ions on $\mathrm{HPAM} / \mathrm{XG}$ supramolecular
3.2.2 The influence of inorganic metal ions on the rheology of CPAM and XG

It can be seen from Figure 4 that the rheology of the mixed solution of cationic polyacrylamide and xanthan gum is poor compared with other ionic or non-ionic polyacrylamide / xanthan gum solutions. when the concentration of monovalent metal ions is increased to $0.1 \%$, it has little effect on the mixed solution of CPAM/XG, and rheology is basically same. When the concentration of monovalent metal ions increases to $0.5 \%$, the shear stress of mixed solution decreased by about $2 \mathrm{~Pa}$, but did not continue to decrease with the increase of concentration. And the effect of divalent metal ions on the mixed solution shows that a small amount of divalent metal ions can reduce the shear stress by about $1 \mathrm{~Pa}$, and with the increase of the concentration of divalent metal ions, the shear stress does not change significantly. 

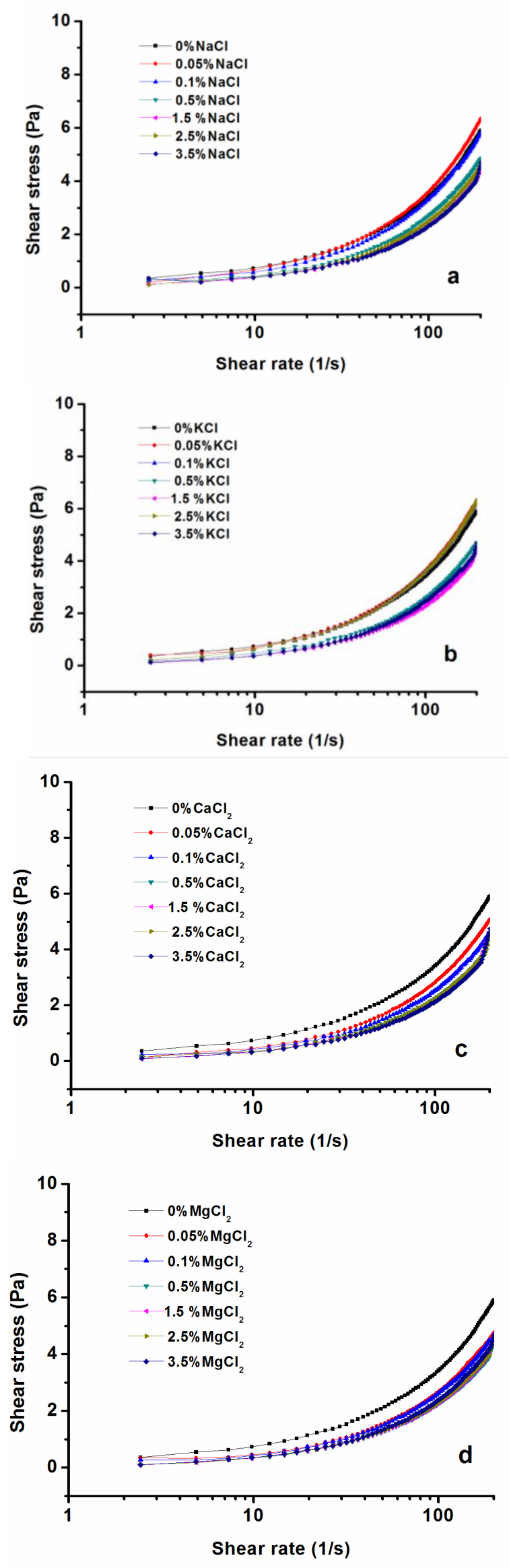

Figure 4. Effect of Inorganic Metal Ions on the Rheology of CPAM and XG Mixed Solution

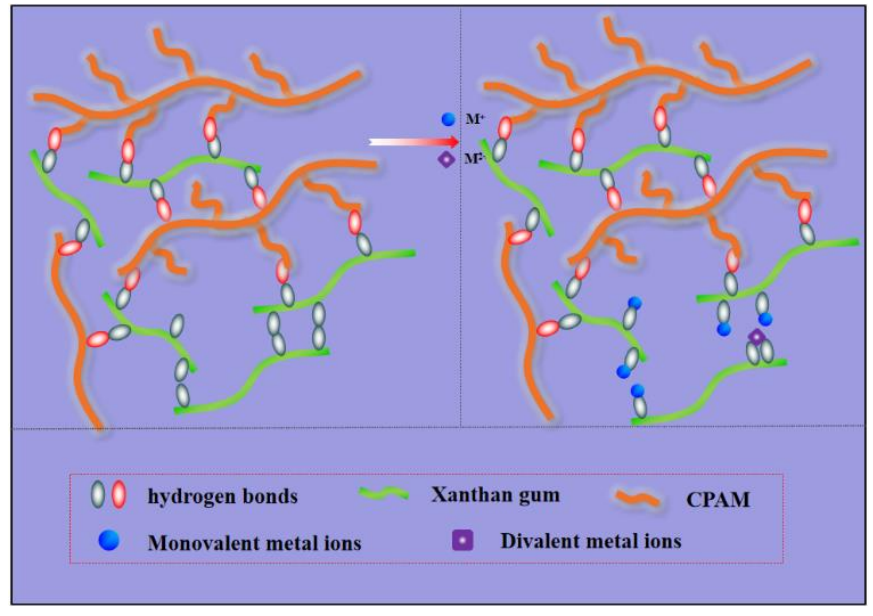

Figure 5. The mechanism of metal ions on CPAM/XG supramolecular

CPAM is a copolymer composed of cationic units and acrylamide non-ionic units. Its molecular chain has positive charge groups that can be ionized (- $\left.\mathrm{CONH}_{2}\right)$, which can be ionized into polycations and small anions in water, it can be adsorbed and bridged with suspended particles dispersed in solution, and has strong flocculation. In Figure 5, when CPAM and $\mathrm{XG}$ are cross-linked, PAM molecules will bridge between particles. In addition to electrostatic attraction between colloidal particles and XG, there are also non-ionic forces such as hydrogen bonds. Although the bonding energy of hydrogen bonds is relatively weak, due to the high degree of $\mathrm{XG}$ polymerization, the total number of hydrogen bonds is also large. Therefore, the strength of the bond is much greater than that of the single electric neutralization, which increases the strength of the floc. When the CPAM/XG mixed solution is interfered by metal ions, the adsorption vacancies on the particle surface decrease and the surface charge of the colloidal particles is reversed, the repulsive force between the particles increases, the bonding bond is weakened, and the adsorption bridging effect between the original particles is weakened, and the viscosity of the mixed solution decreases [15]. However, metal ions can only destroy part of the hydrogen bond between CPAM/XG, but CPAM/XG still has good antiionic stability compared with HPAM/XG mixed solution.

3.2.3 The influence of inorganic metal ions on the rheology of AmPAM and XG

It can be seen from Figure 6 that the mixed solution of zwitterionic polyacrylamide (AmPAM) and xanthan gum (XG) has a very high viscosity. With the addition of monovalent metal ions, its rheological properties also change, and when the concentration of monovalent metal ions is $0.05 \%$, the shear stress can be reduced by $2 \mathrm{~Pa}$, and as the concentration of monovalent metal ions increases, the shear stress of the mixed solution also decreases. When the concentration of monovalent metal ions reaches about $2.5 \%$, the shear stress of the mixed solution remains unchanged. It is obtained that the influence of monovalent metal ions on the mixed solution increases with the increase of metal ion concentration When the concentration of monovalent metal ions reaches a certain level, the salt resistance of xanthan gum has a good inhibitory effect. And it can be seen from Figure $6 \mathrm{c}$ and $6 \mathrm{~d}$ that the $0.05 \%$ concentration of divalent metal ions can make the shear stress of the AmPAM/XG mixed solution drop more, and the drop 
stress reaches $5 \mathrm{~Pa}$. As the concentration of divalent metal ions increases, the shear stress of the mixed solution decreases very little, indicating that a small amount of divalent metal ions can have a greater impact on the rheology of the AmPAM/XG mixed solution, but as the concentration of divalent metal ions increases, the salt resistance of the AmPAM/XG mixed solution The salinity is good and will not have a major impact on the rheology of the mixed solution.

It can be seen from Figure 7 that AmPAM is a high molecular polymer containing anion and cation groups on the macromolecular chain. When AmPAM and XG are in contact with each other, they have a strong affinity. In addition to amide groups, AMPAM contains positive and negative charge groups. Its chain end molecules can stretch in water and bond with XG to form a spatial network structure to enhance water resistance, so the viscosity of the AmPAM/XG mixed solution is relatively large. When the shear rate of the AmPAM/XG mixed solution is low, it will cause chain extension and promote intermolecular association, which will greatly
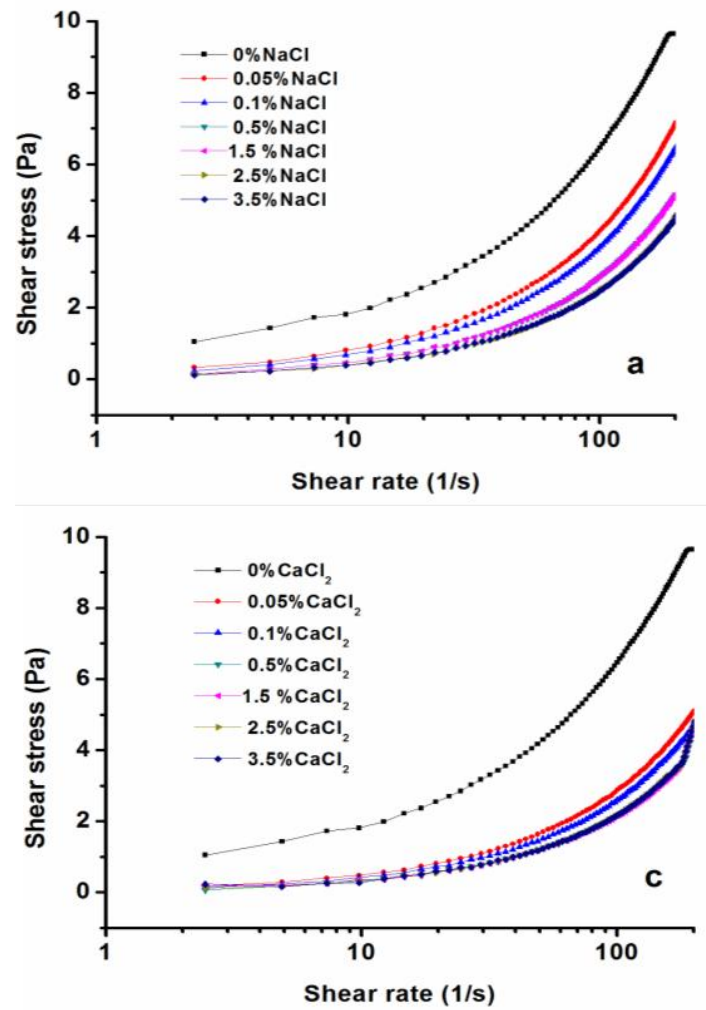

increase the viscosity, when the shear rate is further increased, the intermolecular association structure is damaged when the shear stress is greater than the association force. The shear effect mainly affects the intramolecular and intermolecular association states in the system. In general, viscosity decreases gradually with the increasing of shear rate in dilute solutions. When metal ions are infiltrated into the mixed solution of AmPAM/XG, it will cause molecular chain shrinkage and increase the proportion of intramolecular association [16]. Increasing the proportion of intramolecular association and applying appropriate shear force can offset this shrinkage, and show shear viscosity, but this offset is relative, continuing to increase shear stress will still reduce the viscosity. The rheology of the mixed solution is affected by the ionic degree [17]. The more ionic groups, the better the adsorption and bridging action with metal ions, and the better the flocculation ability. Too many ionic groups reduce the molecular weight of the polymer in the synthesis process, The network structure is weakened in this process, but the flocculation ability decreases.
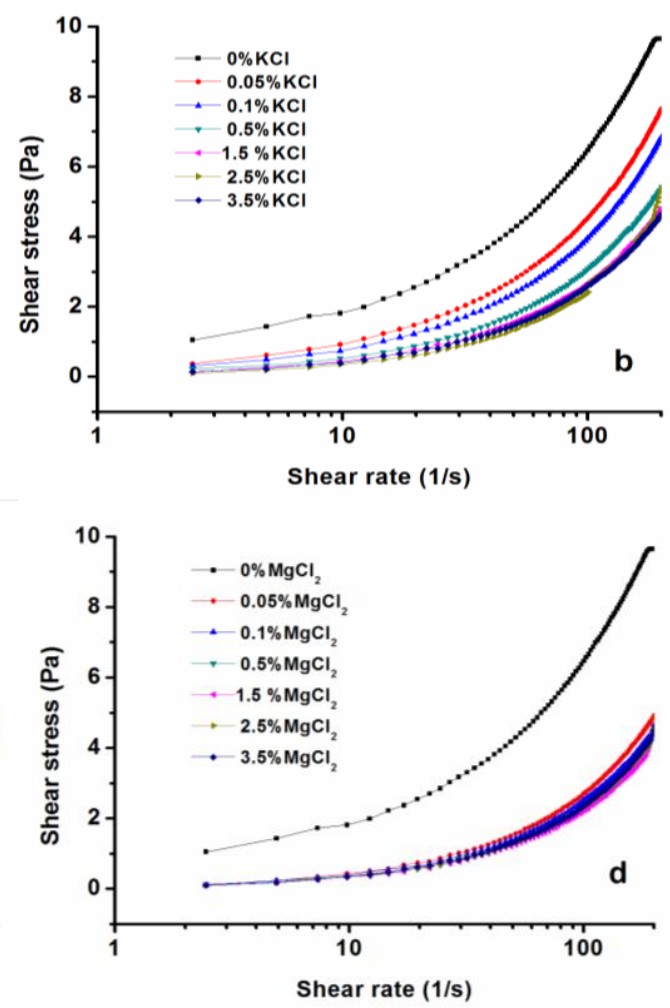

Figure 6. Effect of Inorganic Metal Ions on the Rheology of AmPAM and XG Mixed Solution

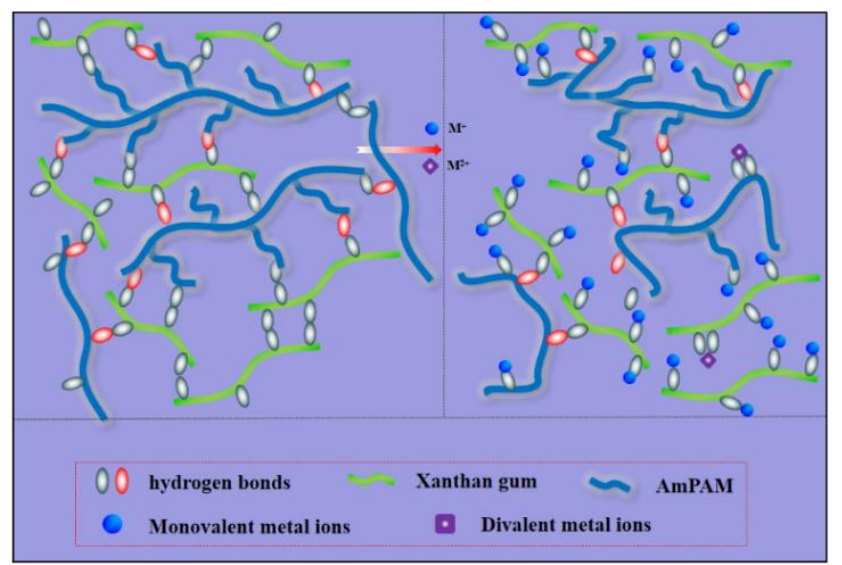

Figure 7. The mechanism of metal ions on AmPAM/XG supramolecular
3.2.4 Effect of inorganic metal ions on the rheological properties of NPAM and XG

In Figures $8 \mathrm{a}$ and $8 \mathrm{~b}$, in the case of $200 \mathrm{~s}^{-1}$, the shear stress of NPAM/XG mixed solution decreases with the increasing concentration of $\mathrm{Na}^{+}$and $\mathrm{K}^{+}$ions. Among them, the order of the effect of $0.05 \%$ concentration of $\mathrm{Na}^{+}, \mathrm{K}^{+}, \mathrm{Ca}^{2+}$ and $\mathrm{Mg}^{2+}$ ions on the shear stress of NPAM / XG mixed solution is $\mathrm{Mg}^{2+}>\mathrm{Ca}^{2+}>\mathrm{Na}^{+}>\mathrm{K}^{+}$, in other words, divalent ions are easier to reduce the viscosity of NPAM / XG mixed solution at high shear rate, that's because non-ionic polyacrylamide is a watersoluble polymer or polyelectrolyte. In Figure 9, when NPAM is in contact with $\mathrm{XG}$, a certain number of polar groups in NPAM molecular chain will form a large spatial network structure with XG through inter particle bridging or charge neutralization. When metal ions are in contact with NPAM / $\mathrm{XG}$ mixed solution, the non-ionic polyacrylamide NPAM is prone to hydrolysis and has anionic negative charge, and the - 
COO- on their molecules is very sensitive to salt, under high salinity and high shear conditions, the viscosity of the aqueous solution will drop significantly [18]. Under the action of electrostatic adsorption [19], the NPAM/XG complex will entrain and bind the metal ions on it, so it will curl, reduce its spatial frame and reduce its viscosity.
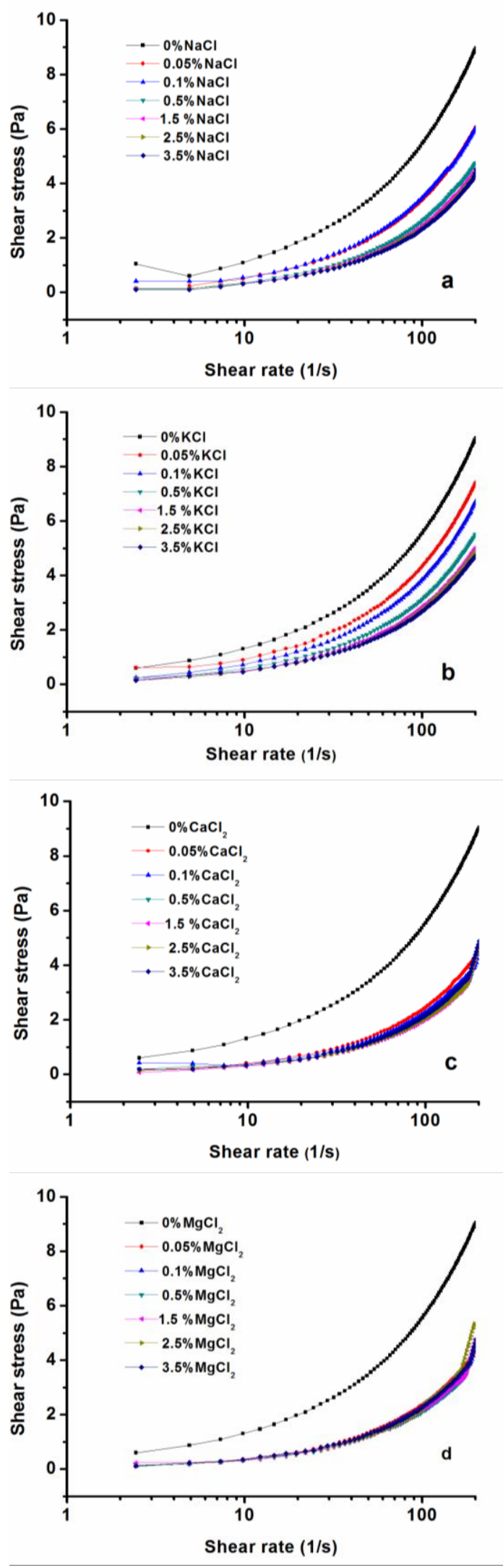

Figure 8. Effect of inorganic metal ions on rheological properties of NPAM and XG mixed solution

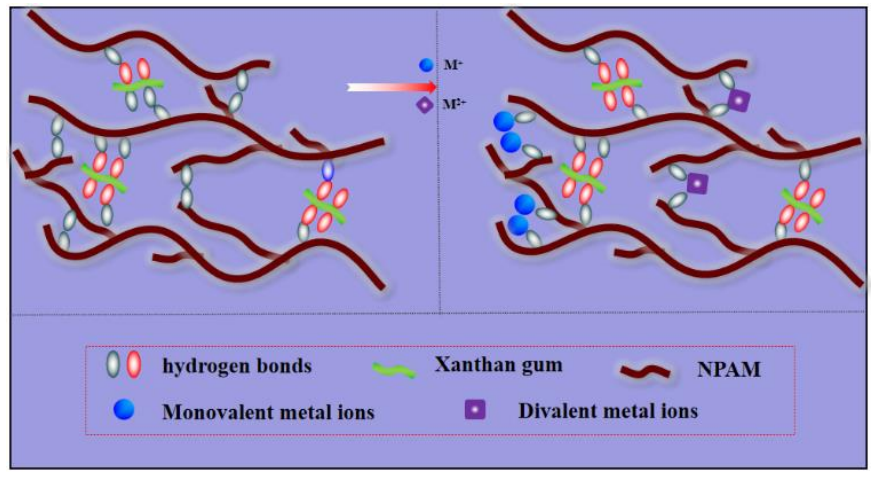

Figure 9. The mechanism of metal ions on NPAM/XG supramolecular

\section{CONCLUSIONS}

Metal cations will destroy the overall viscosity of the $\mathrm{PAM} / \mathrm{XG}$ composite system. Under the same concentration conditions, the destructive power of monovalent ions is less than that of divalent ions. Under the condition of high shear rate, adding the same concentration of metal cations, the shear stress of $\mathrm{CPAM} / \mathrm{XG}$ composite system does not change significantly with the increase of metal ion concentration, and the shear stress of other composite systems changes greatly. The metal ions in the water will destroy its spatial network structure and reduce its foam stabilization time or weaken its viscosity when the PAM/XG conforming system is used as a foam stabilizer for drilling fluids or as a connecting agent for sand-fixation serous.

\section{ACKNOWLEDGEMENTS}

This work was supported by the Jilin Science and Technology Program (Grant No.: 20190303004SF), Jilin Education Program (Grant No.: JJKH20180980KJ), State Key Laboratory of Collaborative Control and Joint Remediation of Soil and Water Pollution under Environmental Protection Open Fund projects (Grant No.: GHBK-2020-021), Changchun Institute of Technology Program (Grant No.: 320210048), College Student Innovation and Entrepreneurship Program (Grant No.: 202111437019).

\section{REFERENCES}

[1] Hu, Z., Wang, D., Zhang, C., Wang, S., Gao, S., Hou, L., Wang, Y. (2019). Diverse noncanonical PAMs recognized by SpCas9 in human cells. bioRxiv, 671503. https://doi.org/10.1101/671503

[2] Jiang, W., Bikard, D., Cox, D., Zhang, F., Marraffini, L. A. (2013). RNA-guided editing of bacterial genomes using CRISPR-Cas systems. Nature Biotechnology, 31(3): 233-239. https://doi.org/10.1038/nbt.2508

[3] Karvelis, T., Gasiunas, G., Young, J., Bigelyte, G., Silanskas, A., Cigan, M., Siksnys, V. (2015). Rapid characterization of CRISPR-Cas9 protospacer adjacent motif sequence elements. Genome Biology, 16(1): 253. https://doi.org/10.1186/s13059-015-0818-7

[4] Leenay, R.T., Maksimchuk, K.R., Slotkowski, R.A., Agrawal, R.N., Gomaa, A.A., Briner, A.E., Beisel, C.L. (2016). Identifying and visualizing functional PAM 
diversity across CRISPR-Cas systems. Molecular Cell, 62(1): $137-147$

https://doi.org/10.1016/j.molcel.2016.02.031

[5] Agoda-Tandjawa, G., Durand, S., Berot, S., Blassel, C., Gaillard, C., Garnier, C., Doublier, J.L. (2010). Rheological characterization of microfibrillated cellulose suspensions after freezing. Carbohydrate Polymers, 80(3):

677-686.

https://doi.org/10.1016/j.carbpol.2009.11.045

[6] Kim, C., Chen, L., Wang, H., Castaneda, H. (2021). Global and local parameters for characterizing and modeling external corrosion in underground coated steel pipelines: A review of critical factors. Journal of Pipeline Science and Engineering, 1(1): 17-35. https://doi.org/10.1016/j.jpse.2021.01.010

[7] Zhu, X.K. (2021). A comparative study of burst failure models for assessing remaining strength of corroded pipelines. Journal of Pipeline Science and Engineering, 1(1): 36-50. https://doi.org/10.1016/j.jpse.2021.01.008

[8] Wang, Z.B., Zheng, Y.G. (2021). Critical flow velocity phenomenon in erosion-corrosion of pipelines: Determination methods, mechanisms and applications. Journal of Pipeline Science and Engineering, 1(1): 63-73. https://doi.org/10.1016/j.jpse.2021.01.005

[9] Zhao, D., Liu, H., Guo, W., Qu, L., Li, C. (2016). Effect of inorganic cations on the rheological properties of polyacrylamide/xanthan gum solution. Journal of Natural Gas Science and Engineering, 31: 283-292. https://doi.org/10.1016/j.jngse.2016.01.047

[10] Akhtar, M., Murray, B.S., Dickinson, E. (2006). Perception of creaminess of model oil-in-water dairy emulsions: Influence of the shear-thinning nature of a viscosity-controlling hydrocolloid. Food Hydrocolloids, 20(6): $839-847$ https://doi.org/10.1016/j.foodhyd.2005.08.006

[11] Alghooneh, A., Razavi, S.M., Kasapis, S. (2018). Hydrocolloid clustering based on their rheological properties. Journal of Texture Studies, 49(6): 619-638. https://doi.org/10.1111/jtxs.12368
[12] Conti-Silva, A.C., Ichiba, A.K.T., Silveira, A.L.D., Albano, K.M., Nicoletti, V.R. (2018). Viscosity of liquid and semisolid materials: Establishing correlations between instrumental analyses and sensory characteristics. Journal of Texture Studies, 49(6): 569577. https://doi.org/10.1111/jtxs. 12358

[13] Garcia-Ochoa, F., Santos, V.E., Casas, J.A., Gómez, E. (2000). Xanthan gum: production, recovery, and properties. Biotechnology Advances, 18(7): 549-579. https://doi.org/10.1016/S0734-9750(00)00050-1

[14] Ghannam, M.T., Selim, M.Y., Zekri, A.Y., Esmail, N. (2019). Flow characteristics of xanthan solutions and their crude oil emulsions in terms of thixotropic behavior. Petroleum Science and Technology, 37(11): 1279-1288. https://doi.org/10.1080/10916466.2019.1566363

[15] Hadde, E.K., Chen, J. (2019). Shear and extensional rheological characterization of thickened fluid for dysphagia management. Journal of Food Engineering, 245:

$18-23$. https://doi.org/10.1016/j.jfoodeng.2018.10.007

[16] Hadde, E.K., Cichero, J.A.Y., Zhao, S., Chen, W., Chen, J. (2019). The importance of extensional rheology in bolus control during swallowing. Scientific Reports, 9(1): 16106. https://doi.org/10.1038/s41598-019-52269-4

[17] Kim, H., Hwang, H.I., Song, K.W., Lee, J. (2017). Sensory and rheological characteristics of thickened liquids differing concentrations of a xanthan gum-based thickener. Journal of Texture Studies, 48(6): 571-585. https://doi.org/10.1111/jtxs.12268

[18] Martín-Alfonso, J.E., Cuadri, A.A., Berta, M., Stading, M. (2018). Relation between concentration and shearextensional rheology properties of xanthan and guar gum solutions. Carbohydrate Polymers, 181: 63-70. https://doi.org/10.1016/j.carbpol.2017.10.057

[19] Sangamithra, A., Sivakumar, V., John, S.G., Kannan, K. (2015). Foam mat drying of food materials: A review. Journal of Food Processing and Preservation, 39(6): 3165-3174. https://doi.org/10.1111/jfpp.12421 